\title{
Project Energize: intervention development and 10 years of progress in preventing childhood obesity
}

\author{
Elaine Rush ${ }^{1 *}$, Carolyn Cairncross ${ }^{1 \dagger}$, Margaret Hinepo Williams ${ }^{1 \dagger}$, Marilyn Tseng ${ }^{2 \dagger}$, Tara Coppinger $^{3 \dagger}$,
} Steph McLennan ${ }^{4+}$ and Kasha Latimer ${ }^{4+}$

\begin{abstract}
Prevention of childhood obesity is a global priority. The school setting offers access to large numbers of children and the ability to provide supportive environments for quality physical activity and nutrition. This article describes Project Energize, a through-school physical activity and nutrition programme that celebrated its 10-year anniversary in 2015 so that it might serve as a model for similar practices, initiatives and policies elsewhere. The programme was envisaged and financed by the Waikato District Health Board of New Zealand in 2004 and delivered by Sport Waikato to 124 primary schools as a randomised controlled trial from 2005 to 2006. The programme has since expanded to include all 242 primary schools in the Waikato region and 70 schools in other regions, including 53,000 children. Ongoing evaluation and development of Project Energize has shown it to be sustainable (ongoing for $>10$ years), both effective (lower obesity, higher physical fitness) and cost effective (one health related cost quality adjusted life year between $\$ 18,000$ and $\$ 30,000$ ) and efficient $(\$ 45 /$ child/year) as a childhood 'health' programme. The programme's unique community-based approach is inclusive of all children, serving a population that is $42 \%$ Mãori, the indigenous people of New Zealand. While the original nine healthy eating and seven quality physical activity goals have not changed, the delivery and assessment processes has been refined and the health service adapted over the 10 years of the programme existence, as well as adapted over time to other settings including early childhood education and schools in Cork in Ireland. Evaluation and research associated with the programme delivery and outcomes are ongoing. The dissemination of findings to politicians and collaboration with other service providers are both regarded as priorities.
\end{abstract}

Keywords: Children, Māori, Indigenous, Physical activity and nutrition, Healthy weight gain

\section{Background}

Over the last decade, the increasing prevalence of obesity and overweight has prompted calls for global action [1] and a global action plan [2]. A recent series in the Lancet [3] addresses the need for global action to impose restrictions on advertising energy-dense and nutrientpoor foods to children, taxes on sugary drinks and

\footnotetext{
*Correspondence: elaine.rush@aut.ac.nz

${ }^{\dagger}$ Carolyn Cairncross, Margaret Hinepo Williams, Marilyn Tseng, Tara

Coppinger, Steph McLennan and Kasha Latimer contributed equally to this work

${ }^{1}$ Child Health Research, Auckland University of Technology, Auckland,

New Zealand

Full list of author information is available at the end of the article
}

regulation of food nutritional quality and availability in schools. A 2011 Cochrane review [4] of interventions for prevention of obesity in children concluded that there was strong evidence of beneficial effects for child obesity programmes on BMI, particularly for children aged 6-12 years. Five of the six promising policies and strategies were centered on schools and the sixth strategy was around parental support and home activities. A recent meta-analysis of school based programmes [5] found that longer interventions ( $>1$ year) were more effective than shorter ones.

While randomised controlled trials are considered the best level of evidence for effectiveness, very little is known about efficacy when interventions are translated 
into real world settings [6]. One of the challenges is that few trials last more than a year; therefore, programme sustainability and ability to maintain effects of the intervention are not known. Feasibility of translation into other settings is also an important research question. In this article, we describe Project Energize, a school-based nutrition and physical activity (PA) programme initiated in the Waikato region of New Zealand in 2003, and key features contributing to its expansion and maintenance. The objective is to describe a programme that has been successful in terms of sustainability and growth (including community and international partnerships), so that others might replicate and adopt similar practices, initiatives and policies.

\section{Project Energize Protocol}

In 2003, the Waikato District Health Board recognised that there was a need to improve the health and wellness of children through co-ordinating a comprehensive, adequately resourced and sustainable Healthy EatingHealthy Living programme that would have long-term benefits. The response was the establishment of Project Energize, a through-school nutrition and PA programme in the Waikato region, delivered by Sport Waikato. Sport Waikato is one of 14 not for profit, regional sports trust in New Zealand who sit under the umbrella of Sport $\mathrm{NZ}$ and work with their communities to get and keep all people physically active and healthy for life. Funding for regional sport trusts come from Sport NZ, government, health providers, funding partners, private organisations and bequests.

The Project Energize programme aims to improve the overall health through increased physical activity and healthier eating and thus reduce the rate of weight gain of Waikato primary school children; of whom 34 \% are Māori [7] and the indigenous people of New Zealand (Aotearoa).

Project Energize began as a quasi-experimental randomized by school cluster, controlled trial of 62 programme schools (including 11,090 children) and 62 matched control schools (10,780 children) between 2004 and 2006 [8]. Following a predetermined randomisation process the 124 schools involved were drawn from 139 who were approached (method paper). Underlying the programme are nine healthy eating goals and seven PA goals that have remained in place since the inception of the programme (Table 1). Wording has changed slightly to incorporate the adaptations made to various national initiatives, such as the National Heart Foundation School Food Programme and Active Schools campaign. The programme is aligned with government food, nutrition and PA guidelines $[9,10]$ and therefore works alongside and with other agencies such as the Heart Foundation. All messages and activities delivered by oral, written and demonstration modes through the programme are composed in ways to be clear, concise and consistent across all intervention components and can be mapped to the programme goals.

The process of engagement with schools followed a sequential pattern of invitations and meetings to present

\section{Table 1 Project Energize healthy eating and physical activity goals}

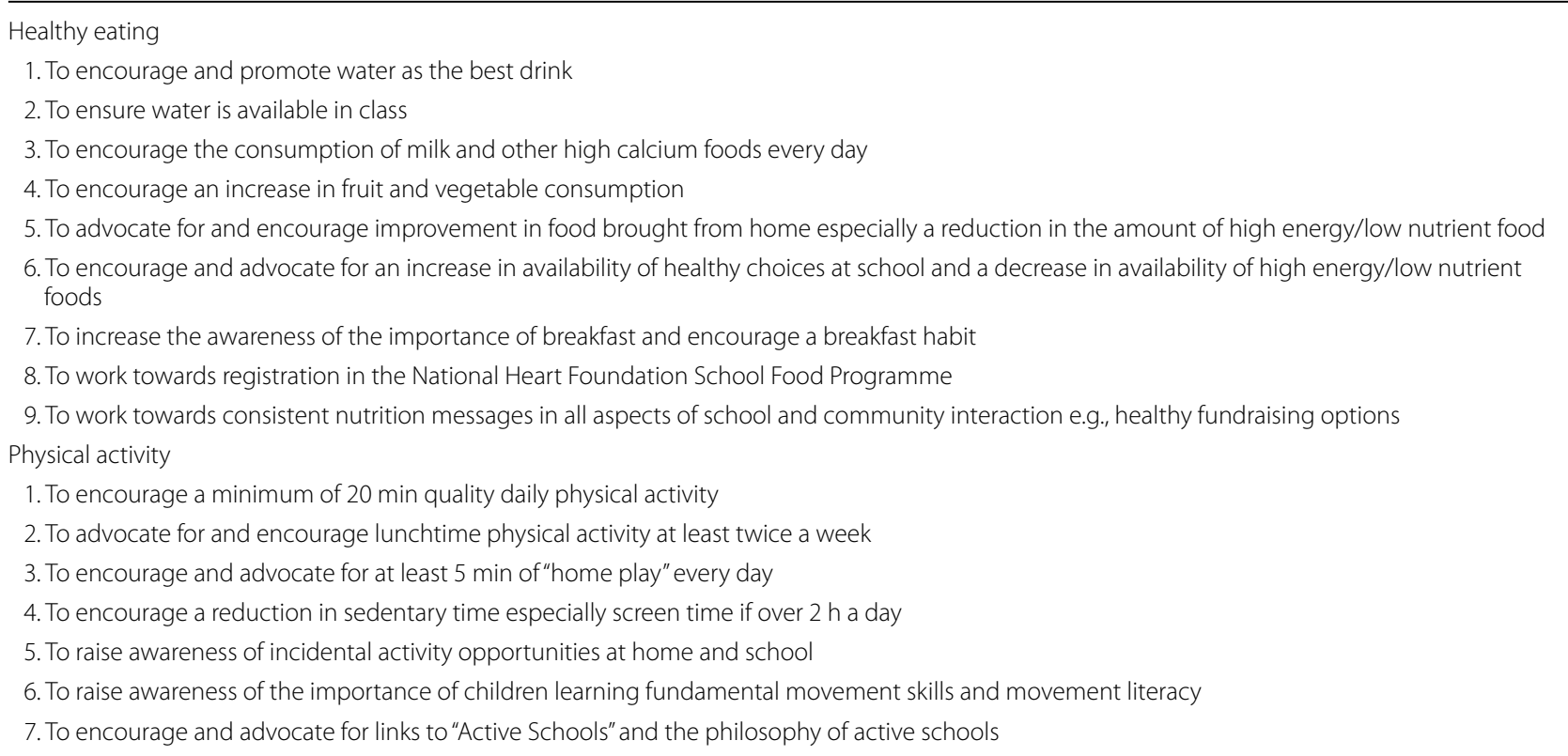


the vision and goals of the programme to the community. At each school a face to face introduction of the programme is provided to school leaders to explain what the programme would mean to them-e.g., one Energizer looking after 6-12 schools in close geographical location and a standard memorandum of understanding signed by the school and a representative of Project Energize. This memorandum explicitly states expectations of who, what and when activities would happen. A lead teacher is appointed as a point of contact for the assigned Energizer. With the Energizer the teacher undertakes a school stocktake detailing what policies, guidelines and practices are currently in place in their school. The current environment for healthy eating and PA is also assessed. From this information, the needs of the school are derived and ranked according to what the school and the Energizer are realistically able to undertake to do in the next year to work towards the goals of the programme. This results in a tailored action plan that is agreed on by the Energizer and the school for one academic year. For example one school may wish to focus on teaching fundamental movement skills (PA goal 5) and another on the quality of food sold at the school canteen (students are not provided with food and often bring lunch from home) and gaining a healthy heart award-healthy eating goal (HE goal 8).

Moreover, the mode of delivery, through a schoolspecific 'Energizer' (a trained PA and nutrition change agent), means that each school receives, in addition to "usual business", a unique, externally-funded service tailored to the needs of that school. The Energizer fields all school interactions, programmes and activities to do with nutrition and PA and is effectively the school go-toperson to ensure that nutrition and PA actions, policies and changes to practice within schools are aligned with governmental goals and are time efficient-for example, delivery of the Kiwi Swim Safe programme by Swimming $\mathrm{NZ}$ to a cluster of schools rather than one by one.

\section{Evaluation}

Measurements of outcomes related to health, knowledge and behaviours of children, parent and staff was conducted according to the guidelines laid down in the Declaration of Helsinki and approved by the Waikato (now Northern Y) Ethics Committee. Both the usual caregiver and the child provided signed informed consent before any measurements were made. The 2004-2006 trial was registered in the Australasian Clinical Trials Registry ACTRN12610000132044.

Findings from the 2004-2006 randomized, by cluster, controlled trial have been published previously and showed evidence with follow-up measures (by external staff) of children (686 boys and 662 girls) aged 5 (1926) and 10 (1426) years (692 interventions and 660 controls) of a reduced accumulation of body fat in younger children, a reduced rate of rise in systolic blood pressure in older children [11], and in a substudy lower vitamin D insufficiency [12] compared with children in control schools. Further, a follow-up evaluation in 2011 of health and fitness outcomes was conducted by Energizers over a six-week period with 24747 year olds and 233010 year olds from 193 of the 243 schools showed improved BMI and run speed outcomes [13] for Energized children compared to historical controls. Data on BMI change measured in the 2011 evaluation [14] were also used as the basis for a lifetime cost effectiveness analysis [15], which concluded that the programme, at $\$ N Z 45 /$ child/ year, was indeed cost-effective from the health treatment payer's perspective, and "would improve quality and length of life when compared with other obesity prevention programmes previously assessed with the same model." Regular audits of Sport Waikato's spending by the Waikato District Health Board show that a large proportion $(>90 \%)$ of the money is spent on the Energizers who are in-the-field and in schools the majority of any school day (personal communication, Sport Waikato management).

\section{Expansion to other schools in NZ}

In 2004, the first 124 schools were approached about the randomized trial, and 62 were randomized to receive the intervention. Since then, the number of schools who have signed up to the programme has increased (Fig. 1). By 2012, all 242 and 44,000 children in Waikato primary schools including special needs schools, were engaged in the initiative, served by 27 Energizers employed by Sport Waikato. Subsequently, in 2013, schools from two other regions in NZ began to receive the Energize programme, with mentorship from the Waikato Energizers and using the resources developed by Sport Waikato (Table 2). In each region, the programme is delivered by the regional sports trust (Sport Waikato, Counties Manukau Sport, Sport Northland) and funded by the sports trust (Counties Manukau Sport) or by the District Health Board (Waikato, Northland), responsible for providing or funding the provision of health services in a given district. In total, Project Energize is being delivered to 53,375 children in 302 schools, or $15 \%$ of all schools in NZ.

As subsequent information collected was for the purpose of implementing a programme delivered through schools rather than for research purposes, approval by an ethics board was not required.

\section{Evaluation of the programme expanded to other settings}

Randomised controlled trials are the cornerstone of proof of efficacious (more good than harm) outcomes, but practically it is not ideal/possible to have children 


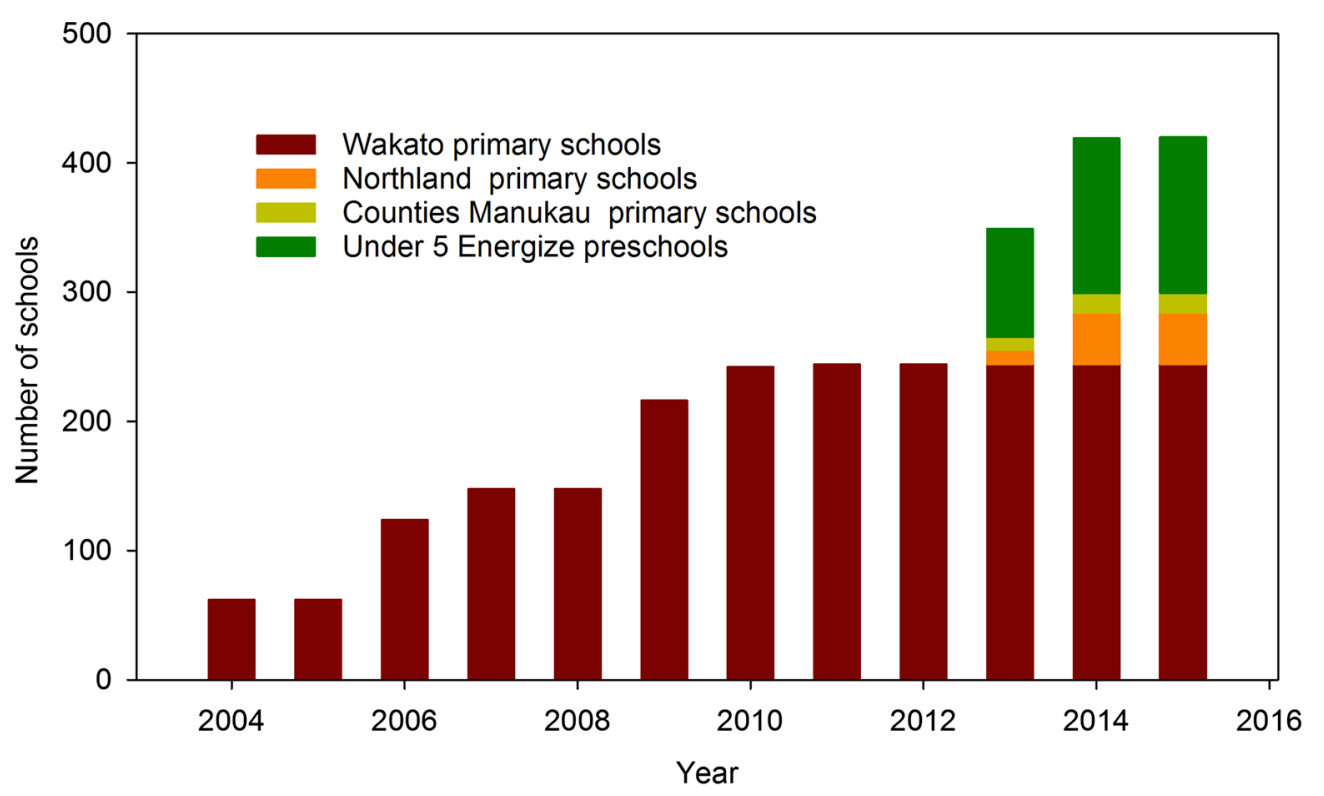

Fig. 1 Progression of engagement of the Project Energize programme with schools

engaged as controls for long periods of time. Further, a continuing challenge [16] with a long-lived programme, is to measure the ongoing effectiveness in the target population. In NZ, parental consent is required to measure children, which is expensive both in time and resources, with the reach of the measures being biased towards those who consent to be measured. It is part of the nature of real world implementation that information collected should be of immediate relevance to the schools themselves.

A primary measure of the effectiveness of Project Energize in the new primary schools is the measurement, before and 2 years after the intervention has been in place, of how fast children can run $550 \mathrm{~m}$. The data are then compared to gender and age-specific run centiles developed from Waikato region data collected in the 2011 evaluation [17, 18]. Run-time is straightforward to measure and in New Zealand does not require parental consent if data are aggregated and de-identified.
Similarly, additional aggregated and de-identified data on food and drink habits and length of time spent undertaking physical education, sport and fitness in the school setting data are collected from new clusters of participating schools via the distribution of standard parent and teacher questionnaires. This information is then returned to the provider and funder at baseline and 2 year followup. As well as informing whether the programme should continue to be supported, this information provides immediate feedback to the Energizers and schools as to where to focus their adaptable action plans e.g., more PA for older classes.

Unlike the original randomized, controlled trial, the review of implementation outcomes [19] is another key component of the continued evaluation of the Energize initiative. Since the inception of the initiative, meticulous documentation, in real time, has enabled feedback to be delivered directly to the funding body (Waikato District Health Board) about the progress of the programme.

Table 2 Number of schools and children receiving the project Energize health service (2014)

\begin{tabular}{|c|c|c|c|c|c|}
\hline Funded by & Delivered by & $\begin{array}{l}\text { Primary schools } \\
\text { (n) }\end{array}$ & $\begin{array}{l}\text { Decile 1-3 } \\
(\%)\end{array}$ & $\begin{array}{l}\text { Children } \\
\text { (n) }\end{array}$ & $\begin{array}{l}\text { Māori } \\
(\%)\end{array}$ \\
\hline Waikato DHB & Sport Waikato & 242 & 32 & 42,000 & 36 \\
\hline Counties Manukau Sport & Counties Manukau Sport & 15 & 33 & 4850 & 25 \\
\hline Northland DHB & Sport Northland & 45 & 100 & 6525 & 71 \\
\hline \multicolumn{2}{|l|}{ Energize total } & 302 & & 53,375 & 42 \\
\hline \multicolumn{2}{|c|}{ NZ totals from ministry of education } & 1961 & 30 & & 23 \\
\hline \multicolumn{2}{|c|}{ Energize as a \% of the NZ total } & 15 & 22 & & \\
\hline
\end{tabular}

Deciles 1-3 are the most socially disadvantaged 
Table 3 Example of a report of frequency of physical activity and healthy eating activities by number of schools, classes and children in 242 Energize schools over 10 weeks by 27 energizers

\begin{tabular}{|c|c|c|c|c|c|c|c|c|c|c|}
\hline Healthy eating (HE goals 1-9) & & 1 & 2 & 3 & 4 & 5 & 6 & 7 & 8 & 9 \\
\hline \multicolumn{11}{|l|}{ \# of schools that ... } \\
\hline Published the Energize nuggets? & 198 & & & & & & & & & $x$ \\
\hline Made healthy changes to their lunch order/canteen? & 15 & & & & & & $x$ & & & \\
\hline Changed their fundraising from unhealthy food & 13 & & & & & & & & & $x$ \\
\hline Signed up for a heart start award this term? & 11 & & & & & & & & $x$ & \\
\hline Were awarded a heart start award this term? & 0 & & & & & & & & $x$ & \\
\hline \multicolumn{11}{|l|}{ \# of classes that ... } \\
\hline Participated in a sugary drinks session? & 22 & $x$ & $x$ & $x$ & & & & & & \\
\hline Participated in a takeaways session? & 18 & & & & & $x$ & $x$ & & & \\
\hline Participated in a breakfast session? & 16 & & & & & & & $x$ & & \\
\hline Participated in a Pro Joe's lunchbox session? & 14 & & & & & $x$ & & & & \\
\hline Participated in a four food groups session? & 13 & $x$ & $x$ & $x$ & $x$ & $x$ & $x$ & & & \\
\hline Participated in a supermarket session? & 2 & & & & & $x$ & & & & \\
\hline Participated in a food for fuel session? & 0 & & & & & $x$ & $x$ & & & \\
\hline Participated in other nutrition sessions? & 9 & & & $x$ & $x$ & $x$ & & & & \\
\hline \# of parents who attended a healthy eating session & 44 & & & & & $x$ & & & & \\
\hline Physical activity (PA goals 1-7) & & 1 & 2 & 3 & 4 & 5 & 6 & 7 & & \\
\hline \multicolumn{11}{|l|}{ \# of schools that ... } \\
\hline Had daily ex sessions modelled? & 59 & & & & $x$ & $x$ & $x$ & & & \\
\hline Had SportsForce workshops? & 49 & & & & & & $x$ & & & \\
\hline Had sports modelled without SportsForce? & 95 & & & & & & $x$ & & & \\
\hline Did some kind of leadership training? & 104 & & & & & $x$ & & $x$ & & \\
\hline Participated in cycling activities? & 45 & & & & & & $x$ & & & \\
\hline Did fundamental movement skills testing? & 12 & & & & & & $x$ & & & \\
\hline Did fitness testing? & 4 & & & & & & & $x$ & & \\
\hline \multicolumn{11}{|l|}{ \# of classes that ... } \\
\hline Did fundamental movement skills testing? & 111 & & & & & & $x$ & & & \\
\hline Did fitness testing? & 17 & & & & & & & $x$ & & \\
\hline \multicolumn{11}{|l|}{ \# of sessions modeling ... } \\
\hline Daily ex & 366 & & & & $x$ & $x$ & $x$ & & & \\
\hline Non-SportsForce & 478 & & & & & & $x$ & & & \\
\hline Other activity sessions & 290 & & & & & $x$ & $x$ & & & \\
\hline Fundamental movement skills testing & 39 & & & & & & $x$ & & & \\
\hline \# of students who participated in cycling activities & 3467 & & & & & & $x$ & & & \\
\hline $\begin{array}{l}\text { \# of teachers who attended a professional development } \\
\text { session }\end{array}$ & 242 & & & & & $x$ & & $x$ & & \\
\hline
\end{tabular}

Each week the Energizers document activities they have undertaken with each school that they work with on to a database. Every term (10 weeks), these reports are collated and sent to the Waikato District Health Board. The report is quantitative and records over 10 weeks the number of schools, classes and children participating in PA and healthy eating activities (Table 3). The activities that are recorded are dependent on each school's individualized action plan. Energizers cannot address every goal with each of their 8-12 schools each term, nor do the schools want to address every goal each term. However, all recorded activities can be linked to one or more of the healthy eating and physical activity goals (Table 1). The report also includes success stories and photographs that the Energizers have assembled about their schools, and these positive success stories are shared with the schools. Documentation additionally involves continuous formative evaluation of what worked and what didn't work during the delivery of the intervention. Such evaluations revealed, for example, that translating resources into 'Te Reo', the Maori language, and starting small with no pressure are approaches that 'work'; that telling schools 
what they should be doing does not work; and that larger schools take longer to implement the programme. Such pragmatic implementation and accountability allows for ongoing modifications to the programme and tailoring for each school, allowing the initiative to continue to strive towards the goal of achieving the improve the overall health through increased physical activity and healthier eating and thus reduce the rate of weight gain, in the most efficient way [20].

\section{Expansion to other populations \\ Under-five age group}

In addition to expansion to a wider geographical area, Project Energize is being adapted and delivered to preschools in four socially disadvantaged areas in the Waikato region. The under 5's intervention follows the same protocol as the original programme but has only six goals; "more active play every day", "milk and water as the best choice". "less sweet drinks", "daily fruit and vegetables", "less energy dense snacks" and "less screen time". Both programmes have the mantra "eat healthy, be active, have fun" which is written on the side of the cars used. In the under 5 Energize programme, four Energizers look after 30 preschools each. This expansion of Project Energize to a pre-school setting is funded by the Ministry of Health and like the original programme, is being delivered by Sport Waikato and evaluated by Auckland University of Technology.

\section{Application in Cork, Ireland}

Project Energize has also been adapted to be piloted in seven intervention $(\mathrm{N}=2089)$ and three control $(\mathrm{N}=1115)$ primary schools in Cork, Ireland. The Irish Energize programme, titled 'Project Spraoi' (pronounced Spree), follows the same protocol and adopts the same goals as the NZ intervention. Only minor adjustments have been made to the programme in order to make it appropriate for an Irish setting, such as adapting activities for play on concrete surfaces instead of grass. Two experienced NZ Energizers visited Ireland prior to the project commencement (August 2013) to provide training and support, and Sport Waikato continues to provide full logistical support for Project Spraoi.

\section{Discussion}

Project Energize has proven to be an effective as well as efficacious school-based programme to combat obesity in children. A unique aspect has been success in maintaining the programme, which celebrated its 10-year anniversary in 2015. With respect to understanding and effecting maintenance and sustainability of a public health intervention programme like Energize, four aspects set it apart from other public health programmes.
First and foremost is its well-trained, stable and knowledgeable workforce [21]. The selection of people for the Energizer role is essential to the ability of the programme to penetrate schools and work with them [22]. Energizers have diverse tertiary qualifications and experience in dietetics, sport, fundamental movement skills, physical education and teaching. The Energizer should live near or within the geographical area that they serve, so that they are seen as part of the community. Moreover, Energizers have secure employment as the programme is a standard budget line for the District Health Board and renewal is part of the annual cycle; the programme is not dependent on the good will of volunteers or local community leaders-an aspect that lends a great deal of stability to the programme.

Secondly, the programme is uniquely sensitive to inequity and to cultural differences. By design, over one-third of the children reached by Energize and 9 of the 27 Energizers are Māori. All resources are translated into Te Reo; methods of delivery consider Māori culture; and Māori games and activities are featured as an integral part of the intervention.

A third feature of the Energize programme that contributes to its continued effectiveness is its accountability. Research and evaluation has been an ongoing characteristic of the quality assurance for the programme, including measures of efficacy as well as of implementation adherence. Immediate feedback from schools to Energizers to Sport Waikato also allow for critical adjustments to programme activities to meet each individual school's needs and enhance its participation.

Finally, successful maintenance of the program is enhanced by community and food environments that support what is modelled in schools-for example, in New Zealand, Fonterra's introduction of free milk to all primary schools that opt-in (https://www.fonterramilkforschools.com/). As another example, in the Waikato region, a few supermarkets also work with Energizers on a weekly "meal deal," where the healthy and simple recipes displayed at the front of the store use ingredients that are currently on promotion. Another supermarket chain offers 'food for thought' (http://www.foodforthought. co.nz) that assists year 5 and 6 primary school students make healthier food and lifestyle choices by providing class resources, nutritionist support and hosting supermarket visits.

The next step for each of the Energize programmes is to further research and document the process of implementation so that similar and future interventions may be informed by this experience. The challenge for Energize is to find ways to be sustainable, to reach the wider community and to work with others to create supportive environments. 


\section{Conclusions}

Project Energize, a cost-effective, community-based, through-school health promotion intervention, has been successfully running in NZ since 2005. It is inclusive of all children and currently involves over 300 primary schools in and beyond the Waikato region. Although other root causes, such as social deprivation and the quality of the commercial food supply, need to be addressed to really have a meaningful effect, it is hoped that the continued rollout of the programme will provide, in the long term, data to show that the prevalence of childhood overweight and obesity is not increasing, particularly in the Waikato region. In the meantime the documentation of the process of implementation and dissemination of Energize will continue to be evaluated, applied and shared with others.

\section{Authors' contributions}

ER conceptualised the manuscript and wrote the first draft. TC provided Ireland content. All authors further developed the concept and reviewed the manuscript. All authors read and approved the final manuscript.

\section{Author details}

${ }^{1}$ Child Health Research, Auckland University of Technology, Auckland, New Zealand. ${ }^{2}$ Kinesiology Department and Center for Solutions Through Research in Diet and Exercise (STRIDE), California Polytechnic University, San Luis Obispo, California, USA. ${ }^{3}$ Department of Sport, Leisure \& Childhood Studies, Cork Institute of Technology, Cork, Ireland. ${ }^{4}$ Sport Waikato, Hamilton, New Zealand.

\section{Acknowledgements}

The authors wish to sincerely thank the teachers, children and their parents for their participation and contributions. The research component was funded by Auckland University of Technology, Sport Waikato, Counties Manukau Sport, Sport Northland, the Northland District Health Board and Waikato District Health Board. The funders of the programme are the Waikato District Health Board, Counties Manukau Sport, Northland District Health Board and the Ministry of Health. The funders had no role in the design, analysis or writing of this article. The programme is currently delivered by Sport Waikato, Counties Manukau Sport and Sport Northland.

\section{Competing interests}

The authors declare that they have no competing interests.

Received: 7 September 2015 Accepted: 8 January 2016

Published online: 26 January 2016

\section{References}

1. United Nations General Assembly. Political declaration of the high-level meeting of the general assembly on the prevention and control of noncommunicable diseases, vol. A/66/L.1. New York: 2011.

2. World Health Organisation. Global action plan for the prevention and control of non-communicable diseases 2013-2020. Geneva: World Health Organisation; 2013.

3. Roberto CA, Swinburn B, Hawkes C, Huang TT, Costa SA, Ashe M, Zwicker L, Cawley JH, Brownell KD: Patchy progress on obesity prevention: emerging examples, entrenched barriers, and new thinking. Lancet. 2015.
4. Waters E, de Silva-Sanigorski A, Hall BJ, Brown T, Campbell KJ, Gao Y, Armstrong R, Prosser L, Summerbell CD. Interventions for preventing obesity in children. Cochrane Database Syst Rev. 2011;(12):CD001871. doi:10.1002/14651858.CD001871.pub3.

5. Sobol-Goldberg S, Rabinowitz J, Gross R. School-based obesity prevention programs: a meta-analysis of randomized controlled trials. Obesity (Silver Spring). 2013;21(12):2422-8.

6. Rothwell PM. External validity of randomised controlled trials: "to whom do the results of this trial apply?". Lancet. 2005;365(9453):82-93.

7. Statistics New Zealand: 2006 census data. 2006. http://www.stats.govt.nz/ Census/2006CensusHomePage.aspx. Accessed 24 Dec 2015.

8. Graham D, Appleton S, Rush E, McLennan S, Reed P, Simmons D. Increasing activity and improving nutrition through a schools-based programme: project Energize. 1. design, programme, randomisation and evaluation methodology. Public Health Nutr. 2008;11(10):1076-84.

9. Ministry of Health, Health Promotion Agency. Eating for healthy children aged 2 to 12/Ngā Kai Tōtika mō te Hunga Kōhungahunga. Revised June 2012. https://www.healthed.govt.nz/resource/eating-healthychildren-aged-2-12ng\%C4\%81-kai-t\%C5\%8Dtika-m\%C5\%8D-te-hungak\%C5\%8Dhungahunga. Accessed 24 Dec 2015.

10. Ministry of Health, Health Promotion Agency. Be active every day: physical activity for 5- to 18-year-olds. December 2010. https://www.healthed. govt.nz/resource/be-active-every-day-physical-activity-5-18-year-olds. Accessed 24 Dec 2015.

11. Rush E, Reed P, McLennan S, Coppinger T, Simmons D, Graham D. A school-based obesity control programme: project Energize. Two-year outcomes. Brit J Nutr. 2012;107(4):581-7.

12. Graham D, Kira G, Conaglen J, McLennan S, Rush E. Vitamin D status of year 3 children and supplementation through schools with fortified milk. Public Health Nutrition. 2009;12(12):2329-34.

13. Rush E, McLennan S, Obolonkin V, Vandal AC, Hamlin M, Simmons D, Graham D. Project Energize: whole-region primary school nutrition and physical activity programme; evaluation of body size and fitness 5 years after the randomised controlled trial. Br J Nutr. 2014;111(2):363-71.

14. Rush E, Graham D, McLennan S, Latimer K. An evaluation of nutrition and physical activity in Waikato primary schools (Project Energize: June 2008 to June 2011). Hamilton: Waikato District Health Board and Ministry of Health, Healthy Eating Healthy Action Evaluation Fund; 2011.

15. Rush E, Obolonkin V, McLennan S, Graham D, Harris JD, Mernagh P, Weston AR. Lifetime cost effectiveness of a through-school nutrition and physical programme: project Energize. Obes Res Clin Pract. 2014;8(2):e115-22.

16. Glasgow RE, Lichtenstein E, Marcus AC. Why don't we see more translation of health promotion research to practice? Rethinking the efficacy-toeffectiveness transition. Am J Public Health. 2003;93(8):1261-7.

17. Rush E, McLennan S, Obolonkin V, Cooper R, Hamlin M. Beyond the randomised controlled trial and $\mathrm{BMI}$ - evaluation of effectiveness of through-school nutrition and physical activity programmes. Public Health Nutr. 2015;18(9):1578-81.

18. Rush E, McLennan S, Obolonkin V, Cooper R, Hamlin M. In response to letter to the editor: beyond the randomised control_-assessment tool. Public Health Nutr. 2015;18:2095.

19. van Nassau F, Singh AS, van Mechelen W, Brug J, Chinapaw MJ. Implementation evaluation of school-based obesity prevention programmes in youth; how, what and why? Public Health Nutr. 2015;18(9):1531-4.

20. Tseng M, Nazmi A. Programmes, policies and implementation. Public Health Nutr. 2015;18(9):1526-7.

21. Yngve A, Tseng M, Haapala I, Hodge A. A robust and knowledgeable workforce is essential for public health nutrition policy implementation. Public Health Nutr. 2012;15(11):1979-80.

22. Mrkusic A. School engagement in the project Energize health intervention programme. 'What works, what does not work, what next?'. Auckland: University of Technology; 2011. 